\title{
The Role of Needle Fenestration with Platelet-Rich Plasma in Chronic Tennis Elbow with Indication of Partial Extensor Tendon Tear
}

\author{
Tracey L Hunter ${ }^{1,2}$, Spencer W Sullivan ${ }^{1 *}$, Struan H Coleman ${ }^{1}$, Eric Brand ${ }^{2}$, James J Kinderknecht ${ }^{1}$ and \\ Vijay B Vad ${ }^{1,2}$ \\ ${ }^{1}$ Department of Sports Medicine, Hospital for Special Surgery, New York, USA \\ ${ }^{2}$ Department of Physiatry, Hospital for Special Surgery, New York, USA

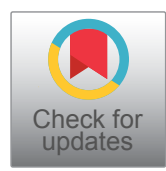

*Corresponding author: Spencer W Sullivan, Department of Sports Medicine, Hospital for Special Surgery, 610 West $58^{\text {th }}$ Street, NY 10019, USA, Tel: 212-774-7810, Fax: 646-885-8252

\begin{abstract}
Objectives: Lateral epicondylitis (LE) is one of the most common elbow conditions in adults. Symptoms of chronic LE can cause severe discomfort and/or physical disability that is economically burdening to patients and health care costs. The purpose of this study is to assess treatment outcomes of pain and function for refractory extensor carpi radialis brevis (ECRB) tendinosis after tendon fenestration with and without platelet-rich plasma (PRP).

Design: Retrospective case series.

Methods: A specialized orthopaedic hospital conducted a retrospective medical chart review of 33 patients from January 1, 2016-December 31, 2017. The study included 27 patients diagnosed with ECRB tendinosis treated with ultrasound-guided ECRB fenestration with $1 \%$ lidocaine/normal saline or PRP. The numeric pain rating scale (NPRS) and Patient-Rated Elbow Evaluation (PREE) were used for quantitative assessment of pain and function.
\end{abstract}

Results: After one lidocaine/saline injection with fenestration (LSIF), $15 \%$ of patients (age mean: $50.5 \pm 8.7$, range: 41-62) with LE showed improvement in pain and function. After two LSIF, $59 \%$ of patients (age mean: 54.8 \pm 5.1 , range: $47-67$ ) with LE showed improvement in pain and function. The patients having a superimposed partial ECRB tendon tear benefited from an additional one LSIF and two leukocyte-poor platelet-rich plasma injections with fenestration (PRPIF), with $26 \%$ of this particular group of LE patients (age mean: $52.9 \pm 5.2$, range: 44-60) showing improvement in pain and function.

Conclusions: Tendon fenestration is safe and effective in $75 \%$ of our patients who failed conservative treatment for
ECRB tendinosis. The presence of a partial ECRB tear is an indicator that fenestration alone was not as effective as a PRP injection with fenestration.

\section{Keywords}

Lateral epicondylitis, Tendinosis, Tennis elbow, Tendon fenestration, Platelet-Rich Plasma, Ultrasound

\begin{abstract}
Abbreviations
LE: Lateral Epicondylitis; ECRB: Extensor Carpi Radialis Brevis; NPRS: Numeric Pain Rating Scale; PREE: Patient-Rated Elbow Evaluation; LSIF: Lidocaine/Saline Injection with Fenestration; PRPIF: Platelet-Rich Plasma Injections with Fenestration; LP-PRP: Leukocyte-Poor Platelet-Rich Plasma; ACD-A: Anticoagulant Citrate Dextrose Solution A
\end{abstract}

\section{Introduction}

Lateral epicondylitis (LE) is a common musculoskeletal condition of the elbow that affects up to $3 \%$ of the general population [1-3]. Acute cases of this joint disorder are usually self-limiting in the young athlete; however, recurring symptoms of prolonged elbow pain and dysfunction tend to occur with debilitating effects beyond adolescence. The pathophysiology of recalcitrant $\mathrm{LE}$ is described as angiofibroblastic tendinosis of the forearm extensor [4-6]. Initial onset typically involves a microtear of the ECRB origin, resulting in painful microtears of tendon.

\footnotetext{
Citation: Hunter TL, Sullivan SW, Coleman SH, Brand E, Kinderknecht JJ, et al. (2021) The Role of Needle Fenestration with Platelet-Rich Plasma in Chronic Tennis Elbow with Indication of Partial Extensor Tendon Tear. Clin Arch Bone Joint Dis 4:013. doi.org/10.23937/2643-4091/1710013

Accepted: February 22, 2021: Published: February 24, 2021

Copyright: (c) 2021 Hunter TL, et al. This is an open-access article distributed under the terms of the Creative Commons Attribution License, which permits unrestricted use, distribution, and reproduction in any medium, provided the original author and source are credited.
} 
Refractory cases, which present in $10-15 \%$ of adults with LE, can be challenging to treat and costly for patient and hospital expenditure [7,8]. Although most patients with recalcitrant LE can be successfully treated non-surgically, the standard of care continues to be clinically debated [9-11]. Chronic LE lacks the acute inflammatory pathogenesis of tendonitis, but rather constitutes tendon degeneration and neovasularity referred to as tendinosis $[3,7]$. The use of corticosteroid injections, anti-inflammatory therapy, should be avoided as it does not target the angiofibroblastic hyperplasia of chronic LE, and long-term use poses significant risk of tendon rupture secondary to impaired collagen metabolism and fibroblast proliferation [12]. As for refractory LE cases requiring surgery, Degen, et al. [13] has shown that $\geq 3$ preoperative injections, particular corticosteroids, increases the chance of revision surgery. Alternative non-conservative interventions for LE include dextrose prolotherapy, extracorporeal shockwave therapy, tendon fenestration, needle tenotomy, botulinum toxin injection, and autologous whole blood or PRP injections.

There is growing demand for use of tendon fenestration and PRP as recent studies have reported positive treatment outcomes for various musculoskeletal conditions, including lateral epicondylalgia and or medial epicondylalgia $[13,14]$ and tendinopathies of the hamstring [15], gluteus medius and minimus $[15,16]$ patella $[17,18]$ and Achilles $[13,19]$ tendons. Tendon fenestration is a needling technique that repeatedly perforates the diseased tendon to induce mechanical reinjury and signal hematological healing processes. Whereas, PRP enhances the delivery of growth factors through increased blood flow to the damaged tendon $[6,20,21]$. Similar to corticosteroid therapy, consideration should be given to the number of pre-operative, therapeutic injections used for LE therapy as 3 or more injections may result in negative outcomes for potential surgical candidates [13].

Our study aims to demonstrate the efficacy of tendon fenestration of the ECRB origin following failure of conservative treatment methods. When ECRB tendinosis presents with a superimposed partial tendon tear, the use of PRP may be an appropriate therapy. Repeat injection may be considered if a single procedure is unsuccessful, as there is potential efficacy in use of multiple PRP injections for chronic tendinopathy [22]. Tendon release surgery may be required if consecutive PRP injections fail; however, in most cases PRP therapy should a promising alterative to surgery for a partial ECRB tendon tear [23]. This study may advance the understanding of percutaneous injection use in tendinosis and contribute to defining the optimal approach and procedure preparations for treating refractory LE.

\section{Methods}

This research study was approved by the institutional review board of Hospital for Special Surgery
(HSS). A retrospective chart review was conducted for 33 patients diagnosed with unilateral ECRB tendinosis during January 1, 2016-December 31, 2017. Selection criteria included patients who failed three months of conservative management, including splinting and/ or counterforce bracing, supervised physical therapy, or 1 corticosteroid injection and had persisting symptoms of pain and/or dysfunction for 3-9 months after LE diagnosis. At the baseline visits, patients were diagnosed with LE based on the clinical assessement and ultrasound imaging performed by a single interventional physiatrist who specializes in sports medicine with over 20 years of experience. If a partial tear was present on ultrasound, MRI confirmation was required to determine the degree of injury. MRI results were assessed by a single, highly-skilled radiologist who specializes in orthopaedic injury and pathology. A low-grade ECRB tendon tear was defined as less than 50 percent torn and a high-grade ECRB tendon tear was greater than 50 percent torn. Patients who experienced significant night pain or demonstrated a positive test for distal, diffuse pain upon palpation of the supinator muscle were suspected to have radial nerve compression. MRI results of these symptomatic patients displayed supinator edema surrounding the entrapped posterior interosseous nerve [24]. Six out of 33 patients diagnosed with ECRB tendinosis with concomitant radial tunnel syndrome were excluded from the study. Final statistical analysis included a total of 27 patients. Data was not stratified or excluded based on patient medical history, including but not limited to diabetes.

All patients with LE who met the study criteria were treated with an ECRB fenestration under ultrasound (Healcerion Sonon $300 \mathrm{~L}$ 5-10 MHz) guidance with lidocaine/saline injection with fenestration (LSIF) [25]. The ECRB tendinosis was visualized on ultrasound by hypoechoic areas indicating characteristic tendon degeneration with signs of hypervascularity on doppler examination $[8,13]$. The needling procedure used a solution of normal saline $(4 \mathrm{~mL})$ and $1 \%$ lidocaine $(4 \mathrm{~mL})$, in which $1 \mathrm{~mL}$ was injected into eight zones of the tendon. If a patient noticed a difference in pain and function after the first injection, but was not completely recovered, the initial procedure was repeated as necessary. In the case when a patient did not experience any symptom relief from fenestration alone, platelet-rich plasma injection with fenestration (PRPIF) was used alternatively, using leukocyte-poor platelet-rich plasma (LP-PRP), for patients until significant improvement occurred. All injection therapies were performed four weeks apart.

After final procedures, all patients were seen for a follow-up appointment at least one year after the baseline visit. The following patient reported outcomes were collected at baseline and follow-up visits: Numeric Pain Rating Scale (NPRS) and Patient Rated Elbow Evaluation (PREE). NPRS uses a standard 0-10 pain ranking $(0=$ no pain; 10 = extreme pain). PREE, which has $95 \%$ test-re- 
test reliability, consists of a series of questions about activities of daily living, included five items on pain and ten items on physical impairment [26]. The items are rated by Likert scales with a scale of $0-10$. The score is determined by adding the 5 points for pain (50) and the weighted total value for the 15 points for function $(150 / 3=50)$ to obtain a total 100 points [26].

The autologous PRP injections were prepared using the Harvest ${ }^{\circ}$ PRP Separation System (Harvest Technologies, Plymouth, MA). Per system instruction, whole blood $(30 \mathrm{~mL})$ was drawn from a peripheral vein using a $35 \mathrm{~mL}$ syringe that contained $3 \mathrm{~mL}$ of Anticoagulant Citrate Dextrose Solution A (ACD-A). The whole blood and ACD-A were transferred into separator chamber containing an additional $1 \mathrm{~mL}$ of ACD-A and underwent double spin centrifugation for a total of 14 minutes at $2500 \pm 150 \mathrm{RPM} \times 1-3$ minutes and $2300 \pm 140 \mathrm{RPM} \times 6-9$ minutes $[27,28]$. The volume of whole blood and ACD-A corresponds to the desired final PRP and the size of the tendon requiring treatment. The LP-PRP preparation required the platelet plasma to be aspirated without either RBC or WBC blood components. The injection site was sprayed with a topical anesthetic, ethyl chloride. Under ultrasound (Healcerion Sonon 300L 5-10 MHz) guidance, the LP-PRP ( $4 \mathrm{~mL}$ ) was injected into the ECRB tendon with fenestrated needling. This technique consisted of an eight-zone needle penetration with $0.5 \mathrm{~mL}$ of LP-PRP injected into each tendon zone. Patients were required to avoid NSAIDs for three days to prevent interruption of platelet growth factors required for tendon healing. Our study assessed the isolated results of PRPIF and was not combined with post-procedure physiotherapy. Post-procedure, patients were instructed to resume regular activity at their own discretion.

\section{Statistical analysis}

Change from preoperative to postoperative outcome scores in the NPRS and PREE were calculated and compared between patients who only received fenestration and patients who also received autologous PRP injections. For changes in NPRS and PREE outcome scores, $95 \%$ confidence intervals $(95 \% \mathrm{Cl})$ were calculated in both cohorts. Two-sample t-tests, assuming unequal variance, were conducted in the reduction of NPRS and PREE using Microsoft Excel 2016 (Microsoft, Redmond, WA). Paired t-tests were calculated between preoperative and postoperative outcome scores in both cohorts. Statistical significance was established at $p<0.05$ a priori.

\section{Results}

After chart review was completed, 27 of 33 patients met the study criteria for the diagnosis of chronic ECRB tendinosis and were included in statistical analysis. Six patients were excluded from the study as they were diagnosed with LE and concomitant radial tunnel syndrome. For comparison of demographic, clinical, and ultrasound measures, the patients were categorized into one of the three following treatment groups: One LSIF, two LSIF, and one LSIF plus two PRPIF. The patient population was $59 \%$ female and $41 \%$ male, with a mean age of 53.9 \pm 5.9 years (range $41-67$ years). The dominant elbow was affected by LE in 22 (81\%) patients (Table 1 ).

Treatment with one LSIF brought significant self-reported relief of pain and disability in four (15\%) patients with LE (age range: 41-62). Treatment with two LSIF brought significant self-reported relief of pain and disability in 16 (59\%) patients with LE (age range: 47-67).

Table 1: Patient demographic characteristics.

\begin{tabular}{|l|l|l|l|l|l|}
\hline & $\begin{array}{l}\mathbf{1} \text { LSIF } \\
\mathbf{( n = 4 )}\end{array}$ & $\begin{array}{l}\text { LSIF } \\
\mathbf{( n = 1 6 )}\end{array}$ & $\begin{array}{l}\text { LSIF only } \\
\mathbf{( n = 2 0 )}\end{array}$ & $\begin{array}{l}\text { LSIF + PRPIF } \\
\mathbf{( n = 7 )}\end{array}$ & Total Population \\
\hline Age (yr), mean (SD) & $50.5(8.7)$ & $54.8(5.1)$ & $53.9(5.9)$ & $52.9(5.2)$ & $53.6(5.7)$ \\
\hline Female, N (\%) & $2(50 \%)$ & $9(56 \%)$ & $11(55 \%)$ & $5(71 \%)$ & $16(59 \%)$ \\
\hline Dominant Elbow, N (\%) & $3(75 \%)$ & $14(88 \%)$ & $17(85 \%)$ & $5(71 \%)$ & $22(81 \%)$ \\
\hline ECRB tendon tear, N (\%) & $0(0 \%)$ & $0(0 \%)$ & $0(0 \%)$ & $7(100 \%)$ & $7(26 \%)$ \\
\hline
\end{tabular}

LSIF: Lidocaine/Saline Injection with Fenestration; PRPIF: Platelet-Rich Plasma Injections with Fenestration; yr: Years; SD: Standard Deviation; N: Number

Table 2: Patient reported outcome measures.

\begin{tabular}{|l|l|l|l|l|l|}
\hline & $\begin{array}{l}\mathbf{1} \text { LSIF } \\
\mathbf{n}=\mathbf{4})\end{array}$ & $\begin{array}{l}\mathbf{2} \text { LSIF } \\
\mathbf{( n = 1 6 )}\end{array}$ & $\begin{array}{l}\text { LSIF only } \\
\mathbf{( n = 2 0 )}\end{array}$ & $\begin{array}{l}\text { LSIF + PRPIF } \\
\mathbf{( n = 7 )}\end{array}$ & Total Population \\
\hline Follow-up (mo), N (\%) & $27.8(1.7)$ & $23.4(5.2)$ & $24.2(5.0)$ & $21.1(4.1)$ & $23.4(4.9)$ \\
\hline Preoperative NPRS, mean (SD) & $6.8(1.3)$ & $8.1(0.9)$ & $7.8(1.1)$ & $7.7(1.1)$ & $7.8(1.1)$ \\
\hline Postoperative NPRS, mean (SD) & $0.3(0.5)$ & $1.9(0.7)$ & $1.6(0.9)$ & $1.9(0.7)$ & $1.6(0.9)$ \\
\hline Preoperative PREE, mean (SD) & $62.5(7.7)$ & $72.3(5.3)$ & $70.3(6.9)$ & $73.3(7.1)$ & $71.1(6.9)$ \\
\hline Postoperative PREE, mean (SD) & $7.5(3.3)$ & $22.3(4.6)$ & $19.3(7.4)$ & $20.6(9.3)$ & $19.6(7.8)$ \\
\hline Conversion to surgery, N (\%) & $0(0 \%)$ & $0(0 \%)$ & $0(0 \%)$ & $1(14 \%)$ & $1(4 \%)$ \\
\hline
\end{tabular}

NPRS: Numeric Pain Rating Scale; PREE: Patient-Rated Elbow Evaluation; mo: Months 
However, LSIF alone failed in seven (26\%) patients and were then treated with one LSIF and two PRPIF. Of the seven patients that underwent PRPIF, $100 \%$ of these patients had LE and a partial tendon tear (age range 44-60). All seven (100\%) of these patients experienced significant self-reported relief of pain and disability. The mean follow-up was $23.4 \pm 4.9$ months (range 15-32 months). Patients treated with only LSIF had an average reduction of $6.3 \pm 0.9(95 \% \mathrm{Cl}=5.879,6.721)$ and $19.3 \pm$ $7.4(95 \% \mathrm{Cl}=48.143,53.851)$ in NPRS and PREE scores, respectively. Patients treated with LSIF plus PRPIF had an average reduction of $5.9 \pm 1.2(95 \% \mathrm{Cl}=4.789,7.011)$ and $20.6 \pm 9.3(95 \% \mathrm{Cl}=46.125,59.275)$ in NPRS and PREE scores (Table 2). The change in NPRS and PREE scores between LSIF alone and LSIF plus PRPIF were not statistically significant ( $p=0.45$ and 0.58 , respectively). However, NPRS and PREE postoperative scores were significantly lower in both cohorts compared to preoperative scores at final follow-up $(p<0.001)$.

Based on ultrasound findings, seven patients were diagnosed with a partial tear of the ECRB tendon. Highgrade tendon tears were found in three female patients. One of the three patients required surgery due to unsuccessful treatment with injection procedures. No full thickness tears were revealed on imaging. All injection procedures were performed without complications.

\section{Discussion}

In this study, we found that ultrasound-guided tendon fenestration is safe and effective in patients who failed conservative treatment for ECRB tendinosis. Collectively, the 20 patients that were treated 1 or 2 fenestrated injections had an average percent reduction of $80 \%$ and $72 \%$ in NPRS and PREE scores, respectively. Interestingly, all of the patients who had a partial tear of the ECRB origin on MR and ultrasound imaging failed treatment with fenestration alone, requiring additional treatment with two PRPIF. The 7 patients in the PRP group experienced an average percent reduction of $76 \%$ and $72 \%$ in NPRS and PREE scores, respectively. Although relief of pain and dysfunction was not significantly different between the patients treated solely with fenestrated injections and patients treated with two PRPIF, it is important to note that all seven (100\%) patients with ECRB tendon tear treated with PRPIF reported significant improvements in pain and function after previous failure of fenestrated injections alone ( $p$ $>0.4$ ).

Although surgery can be highly effective for chronic LE cases, $89-96 \%$ of patients can be successfully treated non-surgically [2]. Additionally, tendon fenestration and PRP has been shown beneficial for tendinosis treatment with fewer adverse risks than corticosteroids and other alternative therapies, such as later, botulinum toxin injection, and extracorporeal shock wave therapy $[10,29,30]$. The present study revealed that one patient with a tendon tear failed PRPIF and underwent surgical tendon repair. All of the patients with a low or high-grade ECRB tear reported a duration of symptoms between 6-12 months (mean: $7.4 \pm 2.6$ ). On average, these patients were burdened by lateral epicondylalgia for longer periods in comparison to the duration of symptoms (range: 3-9 months; mean: $5.3 \pm 2$ ) reported by 12 of 20 patients who did not receive PRP treatment.

According to a few studies, dry needling or saline injections may be efficacious as a standalone therapy in the treatment of chronic LE, but there is medical uncertainty about the indications for ultrasound-guided fenestration with various injected substances (e.g., saline, autologous whole blood, PRP) [31-33]. Stenhouse, et al. [10] conducted a prospective, randomized pilot study assessing the treatment of refractory LE ( $\geq 6$ months) with a two-series injection with autologous concentrated platelets (ACP) or needle fenestration. As replicated in the present study, all injections were performed one month apart. There was greater clinical improvement with ACP at two months following baseline treatment; however, there was no significant difference between the long-term (6 months) benefits of ACP and fenestration. Additionally, Krogh, et al. [34] concluded that injection therapies using PRP or glucocorticoid were not superior to saline for the pain reduction of LE at 3 months. The conclusions from both studies support the value in testing tendon fenestration with saline for ECRB tendinosis without a tendon tear. It is unclear whether the saline or fenestration had independent or synergistic effects in our study; however, this combined injection approach is inexpensive and shown to improve symptoms of unresolved lateral epicondylalgia.

Although limited, peer-reviewed literature found autologous whole blood or PRP superior to tendon fenestration for the treatment of high-grade tendon tears $[12,15]$. The data in the present study promotes a comparable treatment guideline for the use of PRP in both low-grade and high-grade tendon tears in chronic lateral epicondylalgia. Furthermore, Gaspar, et al. [11] concluded that PRP with tendon fenestration has positive outcomes on pain, strength, and function. The patients in this cohort study had a minimum of 6 months of lateral epicondylalgia symptoms and failed treatment with one of the following: Splinting and/or counterforce bracing, supervised therapy, or 1 corticosteroid injection. The follow-up evaluations occurred at a mean greater than 3 years. PRP injections were repeated in 7 of 27 patients who received a baseline treatment with PRP and fenestration due to persistent symptoms. The present study similarly supports Gaspar, et al. [11] in the use of PRP for chronic LE unresolved by prior percutaneous fenestration alone. Although the majority of patients did not require a second procedure in the study by Gaspar, et al. [11] it is important to note that these patients had pre-procedure MR imaging indicating LE without report of a tendon tear. On the contrary, the present study may validate the need for two PRP 
injections in chronic lateral epicondylalgia cases with a partial ECRB tendon tear.

Despite the mixed results of PRP therapies in regenerative medicine, there is growing clinical use of PRP for its benefits in treating tendinopathies. Adequate LE research defends the use of LP-PRP, in particular, for the long-term inflammatory effects that perpetuate tendinosis healing $[7,34,35]$. However, PRP therapies can cost \$500-\$2500, making it a more expensive non-surgical option $[6,36]$. Since the effects of PRP are still not completely understood, health insurance coverage for this procedure is rare. Consequently, PRP treatments are not accessible to all patient populations. Although further research is required, the outcomes of our study can potentially indicate that PRP is unwarranted in the treatment of ECRB tendinosis without a superimposed tendon tear, as needle fenestration alone can be promising and more cost-effective. Conducting further research on autologous blood injections is imperative for making PRP an affordable option when required in the treatment of various musculoskeletal conditions and injuries.

Due to the study design, there are notable limitations. The retrospective nature of this research negates regulation of patient exposure during the treatment period. In addition to missing data for symptom duration of 8 patients, there was a wide variation in the baseline duration of symptoms and patient follow-ups as this could not be standardized. Without comparison to a control group, it is unknown whether the study results would differ from placebo treatment or the wait-andsee approach. The small sample size also resulted in a study analysis that was not powered. This could have affected the statistical significance found between the LSIF alone and the LSIF with PRPIF groups. Considering outcomes of NPRS tend to be subjective, PREE scores provide a standard of general health in elbow pathology with a statistically reliable assessment of joint pain and function [23]. The positive changes for pain and disability outcomes in this study have similarly been reflected in other literature, and therefore, retesting our treatment methods via a multicenter, randomized control study would be valuable. Another strength in this study was the use of ultrasound guidance which provided increased procedure accuracy and a reduced complication rate [26]. Additionally, unlike previous studies, a single interventional physiatrist conducted each fenestration, limiting variability between fenestration techniques.

\section{Conclusion}

This study found that tendon fenestration is safe and effective in $74 \%$ of our patients who failed conservative treatment for ECRB tendinosis. The presence of a partial ECRB tear is an indicator for a PRP injection with fenestration under ultrasound guidance. Overall, this study developed potential parameters for appropriate use of ultrasound-guided PRPIF in tendinopathy while pro- viding insight for injection preparations and needling techniques that have not been clinically defined for chronic lateral epicondylalgia. Therefore, we propose a step-wise approach in the treatment of recalcitrant LE: 1) Physical therapy and exercise; 2) Needle fenestration and 3) Needle fenestration with PRP upon failure to address persistent pain and dysfunction as well as in cases of partial tendon tears. Ultimately, establishing an optimal medical approach for recalcitrant LE would combat related obstacles surrounding treatment and healthcare costs.

\section{Practical implications}

- Ultrasound-guided tendon needling is safe and effective in patients with chronic lateral epicondylalgia.

- A majority of patients reported less pain and increased function after ultrasound-guided tendon needling.

- Patients with a partial elbow tendon tear that failed needle fenestration alone experienced improvements in pain and function after ultrasound-guided tendon needling with platelet-rich plasma injections.

\section{Funding}

This research did not receive any specific grant from funding agencies in the public, commercial, or not-forprofit sectors.

\section{References}

1. Peerbooms JC, Sluimer J, Bruijn DJ, Gosens T (2010) Positive effect of an autologous platelet concentrate in lateral epicondylitis in a double-blind randomized controlled trial: Platelet-rich plasma versus corticosteroid injection with a 1-year follow-up. Am J Sports Med 38: 255-262.

2. Brummel J, Baker CLI, Hopkins R, Baker CLJ (2014) Epicondylitis: Lateral. Sports Med Arthrosc Rev 22: e1-e6.

3. Branson R, Naidu K, du Toit C, Rotstein A, Kiss R, et al. (2017) Comparison of corticosteroid, autologous blood or sclerosant injections for chronic tennis elbow. J Sci Med Sport 20: 528-533.

4. Kaeding C, Best TM (2009) Tendinosis: Pathophysiology and nonoperative treatment. Sports Health 1: 284-292.

5. Bhabra G, Wang A, Ebert JR, Edwards $P$, Zheng $M$, et al. (2016) Lateral elbow tendinopathy: Development of a pathophysiology-based treatment algorithm. Orthop J Sports Med 4: 2325967116670635.

6. Chiavaras MM, Jacobson JA, Carlos R, Maida E, Bentley T, et al. (2014) IMpact of Platelet Rich plasma OVer alternative therapies in patients with lateral Epicondylitis (IMPROVE): Protocol for a multicenter randomized controlled study: A multicenter, randomized trial comparing autologous platelet-rich plasma, autologous whole blood, dry needle tendon fenestration, and physical therapy exercises alone on pain and quality of life in patients with lateral epicondylitis. Acad Radiol 21: 1144-1155.

7. Mishra AK, Skrepnik NV, Edwards SG, Jones GL, Sampson $S$, et al. (2014) Efficacy of platelet-rich plasma for chronic tennis elbow: A double-blind, prospective, multicenter, randomized controlled trial of 230 patients. Am J Sports Med 42: $463-471$. 
8. Enrico C, Marina OR (2018) Ultrasound guided standalone percutaneous needle tenotomy for chronic lateral epicondylitis: A systematic review. Adv Tech Musculoskelet Surg 2.

9. Yoon SK, Thiese MS, Ott U, Kapellusch J, Merryweather A et al. (2019) The role of elbow tender point examination in the diagnosis of lateral epicondylitis. J Occup Environ Med 61: 126-131.

10. Stenhouse G, Sookur P, Watson M (2013) Do blood growth factors offer additional benefit in refractory lateral epicondylitis? A prospective, randomized pilot trial of dry needling as a stand-alone procedure versus dry needling and autologous conditioned plasma. Skeletal Radiol 42: 1515-1520.

11. Gaspar MP, Motto MA, Lewis S, Jacoby SM, Culp RW, et al. (2017) Platelet-rich plasma injection with percutaneous needling for recalcitrant lateral epicondylitis: Comparison of tenotomy and fenestration techniques. Orthop J Sports Med 5: 2325967117742077.

12. Chiavaras MM, Jacobson JA (2013) Ultrasound-guided tendon fenestration. Semin Musculoskelet Radiol 17: 85-90.

13. Degen RM, Cancienne JM, Camp CL, Altchek DW, Dines JS, et al. (2017) Three or more preoperative injections is the most significant risk factor for revision surgery after operative treatment of lateral epicondylitis: An analysis of 3863 patients. J Shoulder Elbow Surg 26: 704-709.

14. Peck E, Jelsing E, Onishi K (2016) Advanced ultrasound-guided interventions for tendinopathy. Phys Med Rehabil Clin N AM 27: 733-748.

15. Suresh SP, Ali KE, Jones H, Connell DA (2006) Medial epicondylitis: Is ultrasound guided autologous blood injection an effective treatment? Br J Sports Med 40: 935-939.

16. Jacobson JA, Yablon CM, Henning PT, Kazmers IS, Urquhart A, et al. (2016) Greater trochanteric pain syndrome: percutaneous tendon fenestration versus platelet-rich plasma injection for treatment of gluteal tendinosis. J Ultrasound Med 35: 2413-2420.

17. Lee JJ, Harrison JR, Boachie-Adjei K, Vargas E, Moley PJ (2016) Platelet-rich plasma injections with needle tenotomy for gluteus medius tendinopathy: A registry study with prospective follow-up. Orthop J Sports Med 4: 2325967116671692.

18. Zayni R, Thaunat M, Fayard JM, Hager JP, Carrillon Y, et al. (2015) Platelet-rich plasma as a treatment for chronic patellar tendinopathy: Comparison of a single versus two consecutive injections. Muscles Ligaments Tendons $\mathrm{J} 5$ : 92-98.

19. James SLJ, Ali K, Pocock C, Robertson C, Walter J, et al. (2007) Ultrasound guided dry needling and autologous blood injection for patellar tendinosis. Br J Sports Med 41: 518-521.

20. Lohrer H, David S, Nauck T (2016) Surgical treatment for achilles tendinopathy-a systematic review. BMC Musculoskelet Disord 17: 207.

21. Gosens T, Peerbooms JC, van Laar W, den Oudsten BL (2011) Ongoing positive effect of platelet-rich plasma versus corticosteroid injection in lateral epicondylitis: A double-blind randomized controlled trial with 2-year follow-up. Am J Sports Med 39: 1200-1208.
22. Tischer T, Bode G, Buhs M, Marquass B, Nehrer S, et al. (2020) Platelet-rich plasma (PRP) as therapy for cartilage, tendon and muscle damage - German working group position statement. J Exp Orthop 7: 64.

23. Hastie G, Soufi M, Wilson J, Roy B (2018) Platelet rich plasma injections for lateral epicondylitis of the elbow reduce the need for surgical intervention. J Orthop 15: 239-241.

24. Raeissadat SA, Rayegani SM, Hassanabadi H, Rahimi R, Sedighipour L, et al. (2014) Is platelet-rich plasma superior to whole blood in the management of chronic tennis elbow: one year randomized clinical trial. BMC Sports Sci Med Rehabil 6: 12.

25. Ferdinand BD, Rosenberg ZS, Schweitzer ME, Stuchin SA, Jazrawi LM, et al. (2006) MR Imaging Features of Radial Tunnel Syndrome: Initial Experience. Radiology 240: 161168.

26. Vincent J, MacDermid JC (2012) The Patient-Rated Elbow Evaluation (PREE). J Physiother 58: 274.

27. Degen RM, Bernard JA, Oliver KS, Dines JS (2017) Commercial separation systems designed for preparation of platelet-rich plasma yield differences in cellular composition. HSS J 13: 75-80.

28. Harvest Terumo BCT (2020) APC Platelet Rich Plasma | Procedure Kits. Harvest Technologies.

29. Galván Ruiz A, Vergara Díaz G, Rendón Fernández B, Echevarría Ruiz De Vargas C (2019) Effects of ultrasound-guided administration of botulinum toxin (incobotulinumtoxina) in patients with lateral epicondylitis. Toxins 11: 46.

30. Haake M, Böddeker I, Decker T, Buch M, Vogel M, et al. (2002) Side-effects of extracorporeal shock wave therapy (ESWT) in the treatment of tennis elbow. Arch Orthop Trauma Surg 122: 222-228.

31. McShane JM, Shah VN, Nazarian LN (2008) Sonographically guided percutaneous needle tenotomy for treatment of common extensor tendinosis in the elbow. J Ultrasound Med 27: 1137-1144.

32. Sorani A, Campbell R (2015) Image-guided elbow interventions: A literature review of interventional treatment options. Br J Radiol 89: 20150368.

33. Krey D, Borchers J, McCamey K (2015) Tendon needling for treatment of tendinopathy: A systematic review. Phys Sportsmed 43: 80-86.

34. Krogh TP, Fredberg U, Stengaard Pedersen K, Christensen $R$, Jensen $P$, et al. (2013) Treatment of lateral epicondylitis with platelet-rich plasma, glucocorticoid, or saline: A randomized, double-blind, placebo-controlled trial. Am J Sports Med 41: 625-635.

35. Dragoo JL, Braun HJ, Durham JL, Ridley BA, Odegaard JI, et al. (2012) Comparison of the acute inflammatory response of two commercial platelet-rich plasma systems in healthy rabbit tendons. Am J Sports Med 40: 1274-1281.

36. Le ADK, Enweze L, DeBaun MR, Dragoo JL (2018) Current Clinical Recommendations for Use of Platelet-Rich Plasma. Curr Rev Musculoskelet Med 11: 624-634. 